\title{
Morphogenesis of an Institution on a Lattice Game
}

\author{
ELETTRA AGLIARDI ${ }^{\mathrm{a}, *}$ and EMANUELE GIOVANNETTI ${ }^{\mathrm{b}}$ \\ a Department of Economics, University of Bologna, Piazza Scaravilli 2, \\ 40126 Bologna, Italy; ' Department of Economics, University of Rome, Rome, Italy
}

(Received 2 February 1998)

\begin{abstract}
In this paper we study the morphogenesis of an institution when local social interactions are taken into account. The structure we obtain has characteristics of "self-organized criticality". After a transient period the system self-organizes into a configuration which is compatible with a high degree of differentiation among different sites and generates typical power laws.
\end{abstract}

Keywords: Institutions, Self-organization, Differentiation

\section{INTRODUCTION}

In abstract terms institutions can be defined as orderly and more or less persistent behaviour patterns, "rules or set of rules that constrain or govern organized patterns of behaviour" (Langlois, 1986). These patterns may emerge spontaneously, that is unintentionally, or may result from consciously designed rules of interactions.

In this paper we study the morphogenesis of an institution when local social interactions are taken into account. While much of the analysis of how institutions emerge and change over time has been developed within coordination and common interest games and has been mainly concerned with the evolution of conventions (see, among others, Ellison, 1993; Mailath, 1993; Young, 1993), in this paper we study the morphogenesis of an institution, that is, the space-time pattern of the evolution of the players' actions, considering an "anti-coordination" payoff structure, focusing on local interactions and applying different techniques.

We study a lattice game in which some degree of stability is reached over time after agents have tuned their actions to the moves of their neighbour agents. The main result of the paper is that the structure we obtain has characteristics of "selforganized criticality" (Bak and Chen, 1991; Bak et al., 1988). After a transient period the system selforganizes into a configuration which is compatible with a high degree of differentiation among different sites and generates power laws for the behaviour of objects such as spatial correlation functions. Notice that with the exception of Bak et al. (1993) there are no papers applying the model of "self-organized criticality" to economic

* Corresponding author. 
problems. Their paper, however, differs from ours because we consider a different lattice structure, a different specification of the random shocks and a different source of local interaction.

The structure of the model is presented in Section 2; Section 3 contains the main results and Section 4 concludes with final remarks.

\section{THE MODEL}

The model focuses on a dynamic context with the following features. We consider $N$ agents arrayed on a one-dimensional circular lattice at a unitary discrete distance, where the agents' indices $i$ denote locations. Neighbours of $i$ are denoted by indices $i-1$ and $i+1$. The circular lattice implies that the left neighbour of agent 0 is agent $N-1$ and the right neighbour of agent $N-1$ is agent 0 . Each agent chooses one of the two possible actions, 0 or 1 , at every period $t, t=1,2, \ldots$, and choices are temporarily irreversible, that is, switching is not allowed within each period. We assume that the payoff earned by any particular agent $i$ at $t$ only depends on his current action and those chosen by his two immediate neighbours $i-1$ and $i+1$. Payoffs are assumed to reflect "local substitutability", that is, if an agent has both of his neighbours choose a given action, then the best for him is choosing the alternative one. Table I specifies agent $i$ 's payoffs in our "anti-coordination" game.

In Table I the choice of action 0 yields payoff 0 . If agent $i$ chooses action 1 and there is no

TABLE I

\begin{tabular}{lccc}
\hline & Actions & & i's payoff \\
\cline { 1 - 2 }$i-1$ & $i$ & $i+1$ & \\
\hline 0 & 1 & 0 & 2 \\
0 & 1 & 1 & 1 \\
1 & 1 & 0 & 1 \\
1 & 1 & 1 & -1 \\
0 & 0 & 0 & 0 \\
0 & 0 & 1 & 0 \\
1 & 0 & 0 & 0 \\
1 & 0 & 1 & 0 \\
\hline
\end{tabular}

competition by the two neighbours, then $i$ 's payoff is 2 , while if there is just one competitor, $i$ 's payoff is 1. In the case of competition by both neighbours agent $i$ loses 1 . The game can be interpreted in terms of technological competition where action 1 corresponds to adoption of an innovation, or alternatively, in terms of entry in a market which reduces the equilibrium price, or diffusion of new ideas and scientific knowledge, etc., where "strategic substitutability" is local.

At $t$ each $i$ is subject to the realization of a random variable $\mu$ uniformly distributed in $[0,1]$. The agent with the highest realization, say $i^{*}$, moves first and chooses his best possible action. (In this framework receiving the highest shock means that the agent, randomly selected, has had the most successful idea, "mutation", from which a superior technology, a piece of knowledge, a new pricing policy stem.) Other agents then move subsequently within this period choosing action 1 or 0 depending on the actions and payoffs of each of their neighbours. As an example, suppose we start from an initial condition characterized by actions equal to 0 for all agents and therefore by 0 payoffs for all agents. The agent with the highest prevailing draw will choose action 1 and therefore receive a payoff equal to 2 , while the others continue to earn 0 . The higher payoff earned by $i^{*}$ is assumed to be locally observable by the two neighbours $i^{*}-1$ and $i^{*}+1$ who will find it profitable to imitate $i^{*}$ 's action. Reorganization in the payoffs levels occurs as from Table I. For example, if at the end of period $t$ only $i^{*}-1$ and $i^{*}+1$ are imitators, they will get a payoff equal to 1 each, while $i^{*}$ will get -1 as a result of competition with cumulative payoffs equal to 1 for all of them (see Table II).

The idea reflected here is that an agent will imitate the action adopted by one of his neighbours

TABLE II

\begin{tabular}{rrrrrrrrrrrrrrrr}
\hline \multicolumn{11}{c}{ Actions } & \multicolumn{11}{c}{ Payoffs } \\
\hline 0 & 0 & 0 & 0 & 0 & 0 & 1 & 0 & 0 & 0 & 0 & 0 & 0 & 0 & 2 & 0 \\
0 & 0 & 0 & 0 & 0 & 1 & 1 & 1 & 0 & 0 & 0 & 0 & 0 & 1 & -1 & 1 \\
& & & & & & & & 0 & 0 & 0 & 0 & 0 & 1 & 1 & 1 \\
\hline
\end{tabular}


if the latter's payoff is sufficiently larger than his own. That is, agents will adopt the following "learning rule": If one of their neighbours' cumulative payoff is $\geq 2$ than their own, then they will find it profitable to imitate that neighbour's action; otherwise, they will stick with the action they did in the past.

At the end of period $t$ we have a set of agents which have increased their level of payoffs while the complement of this set has not registered any change. Then period $t+1$ starts. We assume that new random noise $\mu^{\prime} \in[0,1]$ is assigned only to all adjusted sites. This implies that if the vector of random shocks in the first period was $\mu=\left\{\mu_{i}\right.$ for $i=1,2, \ldots, N\}$, in the following period the new random vector $\mu^{\prime}$ will have new components $\mu_{i}^{\prime}$ again uniform in $[0,1]$ only for the sites which have changed action, while the other components remain unchanged. Once the new vector of random independent draws is derived, the process is repeated choosing the site which has the new highest draw and starting a new round of reorganization, assigning payoffs as from Table I.

Obviously, the "learning rule" we introduced above acts as a crucial element in the process of transmission in this system: Through the chain of neighbours the effects of local externalities may spread along long distances too, until reorganization in the payoff levels stops. The distribution of payoffs represents the distribution of property rights and we are interested in asking how property rights do evolve.

\section{A SELF-ORGANIZED CRITICAL SYSTEM: SIMULATIONS RESULTS}

In this section we study the statistical properties of our model. First, we analyse the features of the distribution of the random shocks. Observe that in this setting the probability distribution of the random shocks changes with time. The substitution of the highest prevailing draw and all the other values of random draws of the sites which revised their actions with new random realizations while keeping the other components unchanged modifies the probability distribution for each site of being selected as the site with the new highest random shock as a function of the history of previous realizations of the shocks. The features of the random mechanism are such that at the beginning subsequent events are quite uncorrelated in space but after a transient period the distribution of the highest draws becomes stationary and events become correlated. Following Bak and Sneppen (1993) we can measure the distribution of the distance between subsequent sites in which actions are revised. Figure 1 shows indeed that the system will reach a state where the distribution of the distances between subsequent sites with highest shocks can be described by a power law distribution. This does not depend on the initial condition, so the critical state is a global attractor for the dynamics. Observe that this result is expected within a model of "self-organized criticality". We recall here that the model of self-organized criticality has been proposed recently by Per Bak and his colleagues at the Brookhaven National Laboratory in the USA and has been studied mainly through computer simulations and controlled experiments. Their paradigm is a computer model of a "sand pile", built from uniform square grains stacked on top of each other in columns, where grains are added to the pile, one at a time, onto the tops of the columns and the locations for the added grains are chosen at random so that the pile grows with an uneven surface. When the height difference between neighbouring columns reaches some predetermined threshold the surface grains

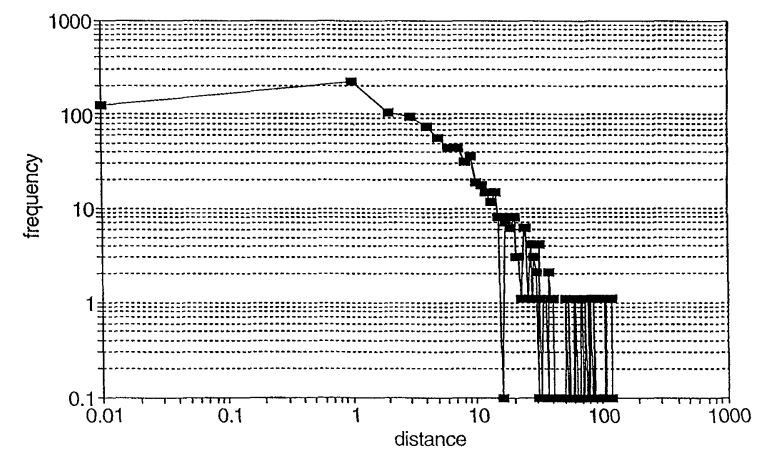

FIGURE 1 Frequency distribution of the distances. 
flow, or fall, from the higher to the lower column. In turn, this movement may leave other neighbouring columns in an unstable condition so that further surface flow could occur. The computer model completes all the surface movements in a chain or avalanche process, before any more grains are added.

In Fig. 2 we have obtained the surface representing the distribution of cumulative payoffs for a system consisting of 65 players after 10100 runs of the game described in Section 2. Figure 2 represents the snapshot of a time-space stochastic process which starts with no asymmetries among players and, after a transient period, generates a highly asymmetric distribution of the payoffs in space. What is particularly interesting is the endogenous emergence of localized clusters of "successful" players, intermitted by less "successful" ones. Such configuration, which is characterized by a high degree of differentiation among different sites, can be interpreted as the market configuration resulting from the processes of diffusion of knowledge, technological competition, pricing policies, etc., described by the game in Section 2. Moreover, both an accurate analysis of the surface and the study of the standard deviation of the cumulative payoffs reached in the different sites as a function of the system size (Fig. 3) show the presence of fractal characteristics and self-affinity. One of these features is the roughness of the surface expressed

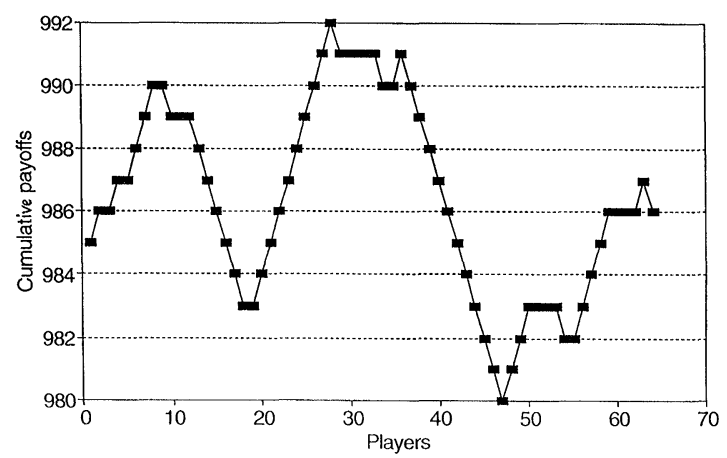

FIGURE 2 Cumulative payoffs distribution (after 10100 runs of the game). by means of the standard deviation of the cumulative payoffs among sites. The roughness increases with the number of players following a power law, which means that the logarithmic transformation of the standard deviation increases linearly with the logarithm of the number of players, with a coefficient equal to 0.73 . This is represented in Fig. 3; the standard deviation has been calculated starting from the cumulative payoffs for a number of players ranging from 50 to 1000 players expressed by

$$
\operatorname{std}(N)=\sqrt{\frac{1}{N} \sum_{i=1}^{N}\left(\Pi_{i, t}-\tilde{\Pi}_{t}\right)^{2}},
$$

where $\tilde{\Pi}_{t}=\left(\sum_{i=1}^{N} \Pi_{i, t}\right) / N$ and $\Pi_{i, t}$ is the cumulative payoff of player $i$ at time $t$.

Power law relations among variables are also interpreted as signs of a self-organized critical state. The dynamics of the spatio-temporal stochastic process describing the evolution of cumulative payoffs in time and among sites seems to converge towards a configuration which is continuously changing but which preserves certain features, like its roughness on all space-scales, unchanged. From Fig. 4 we can analyse the typical aggregate variability in the system at each period for 64 players after 10100 repetitions of the game described in Section 2. The aggregate variability,

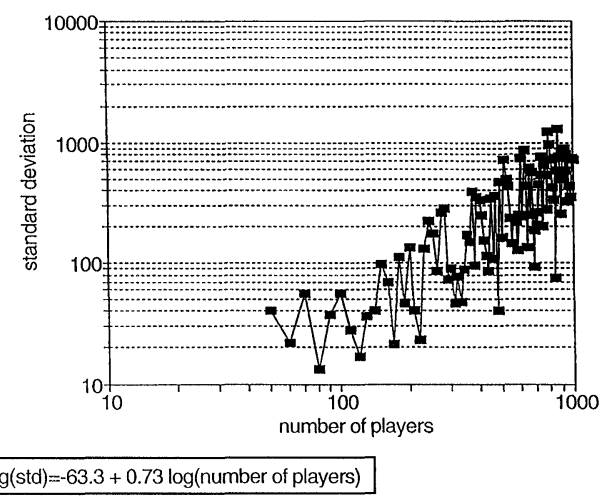

FIGURE 3 Standard deviation versus system size (from 50 to 1000 players, 10000 games). 


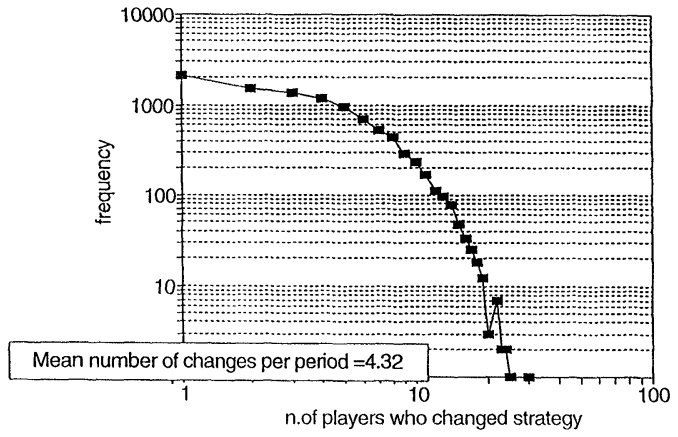

FIGURE 4 Distribution of strategy changes (after 10100 runs with 64 players).

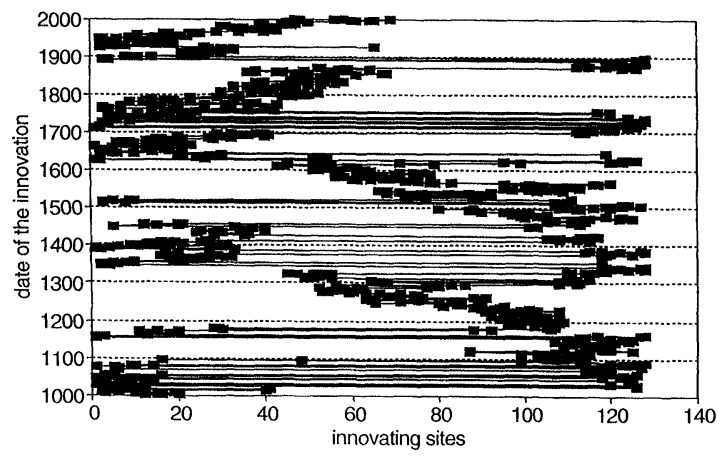

FIGURE 5 Localization of the innovations (1000 periods).

that is, the numerosity of the sites which change actions, remains constant over time with a characteristic size of 4.32 . The number of players which change actions and therefore register a change in payoffs defines the size of an "avalanche" and depends on the previous period configuration and the shocks realization. Then Fig. 4 represents the distribution of the sizes of avalanches for this model, where the mean avalanche size is 4.32 : The distribution of the size of activity does decline exponentially, therefore not showing power law type of behaviour.

Finally, Fig. 5 shows the intermittency of the evolution of the sites in which actions are revised. We plot the date of a revision against the number of revisions and observe long periods of passivity punctuated here and there by sudden bursts of activity: Thus, the model exhibits "punctuated equilibria" (Gould and Eldredge, 1993).

\section{FINAL REMARKS}

By explicitly recognizing the role of local structure on the gobal properties of an economic system a rich class of models can be developed. The approach and model of morphogenesis outlined above, although simplified, suggests a potentially fruitful direction where research could be addressed. The analysis of the spatio-temporal pattern of the evolution of the agent's action seems to us important to understand how agents interact and learn in path-dependent systems and how economic institutions and social norms evolve. Our model is just one step towards understanding the general phenomenon of the effect of local structure on global properties.

\section{References}

Bak, P. and Chen, K. (1991) Self-organized criticality, Scientific American, 264, 46-51.

Bak, P., Chen, K., Scheinkman, J. and Woodford, M. (1993) Aggregate fluctuations from independent sectoral shocks: selforganized criticality in a model of production and inventory dynamics, Ricerche Economiche, 47, 3-30.

Bak, P. and Sneppen, K. (1993) Punctuated equilibrium and criticality in a simple model of evolution, Physical Review Letters, 71, 4083-6.

Bak, P., Tang, C. and Wiesenfeld, K. (1988) Self-organized criticality, Physical Review A, 38, 364-78.

Ellison, G. (1993) Learning, local interaction and coordination, Econometrica, 61, 1047-71.

Gould, S.J. and Eldredge, N. (1993) Punctuated equilibrium comes of age, Nature, 366, 223-7.

Langlois, R.N. (1986) Economics as a Process (Cambridge, Cambridge University Press).

Mailath, G.J. (1993) Perpetual randomness in evolutionary economics, Economics Letters, 42, 291-9.

Young, H.P. (1993) The evolution of conventions, Econometrica, 61, 57-84. 


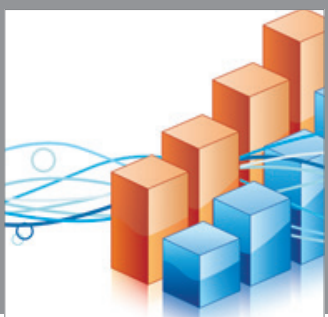

Advances in

Operations Research

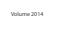

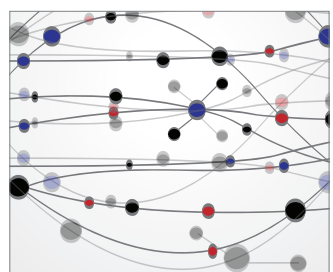

\section{The Scientific} World Journal
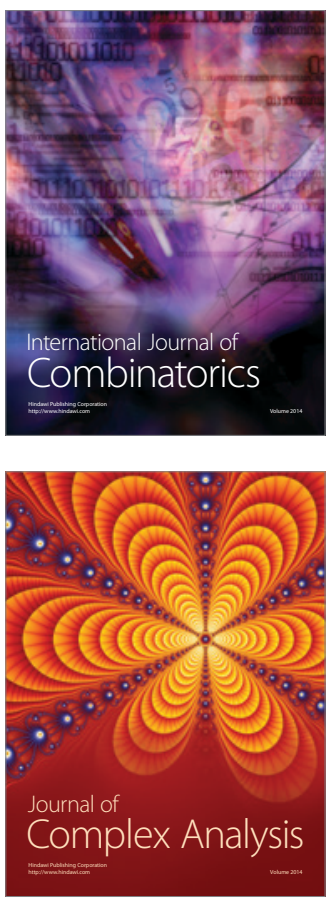

International Journal of

Mathematics and

Mathematical

Sciences
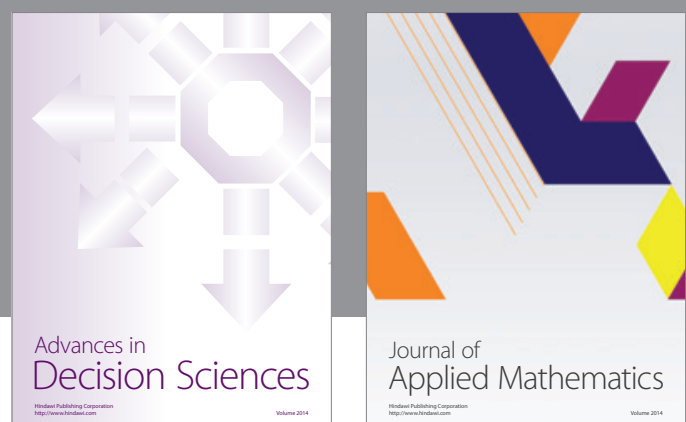

Journal of

Applied Mathematics
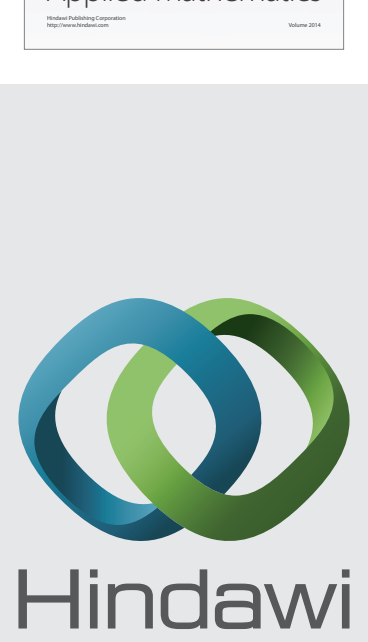

Submit your manuscripts at http://www.hindawi.com
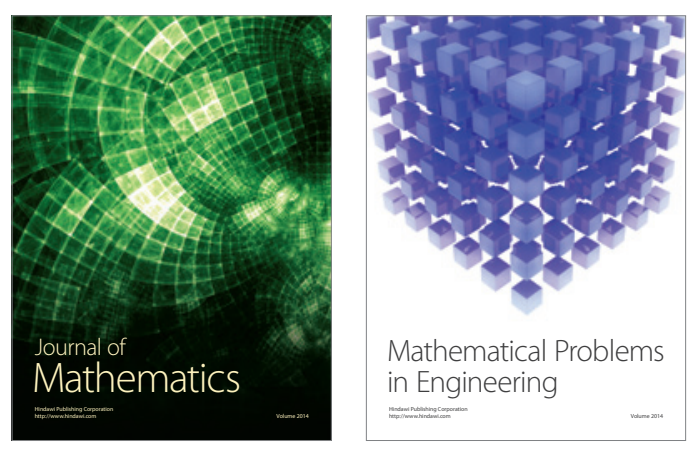

Mathematical Problems in Engineering
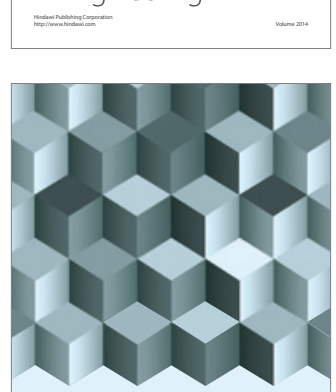

Journal of

Function Spaces
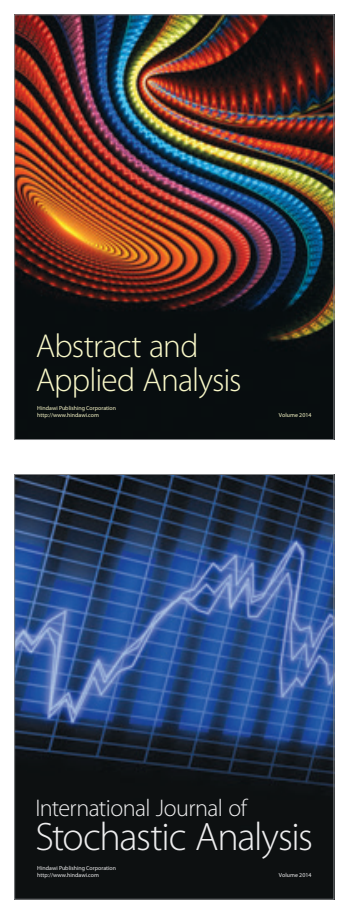

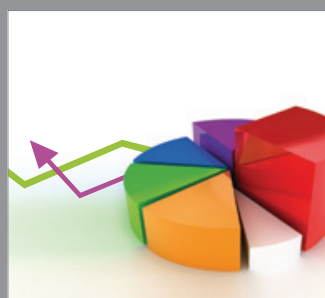

ournal of

Probability and Statistics

Promensencen
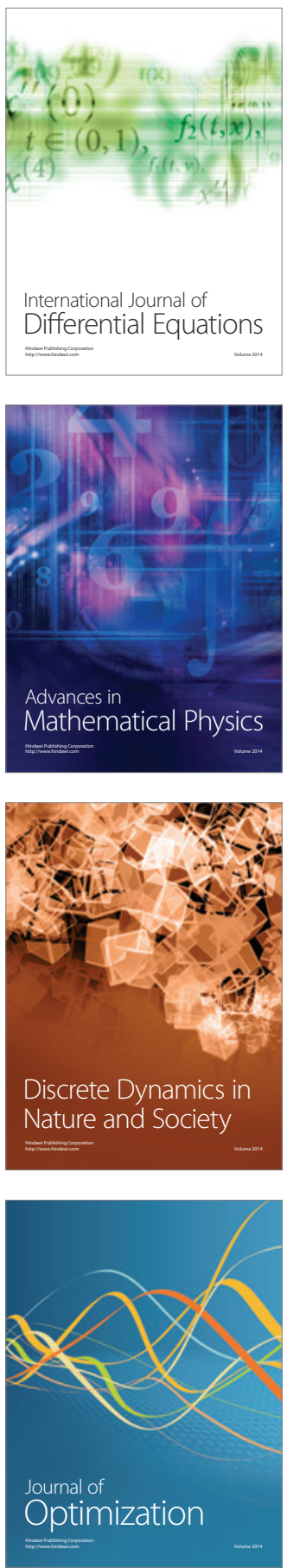